\title{
Einfluß demographischer Änderungen auf Fallzahlen und Pflegetage hospitalisierter internistischer Patienten
}

\author{
T. Mansky, C. M. Erben, S. Kleinschmidt, H. Fassl und P. C. Scriba \\ Zentrum Innere Medizin ${ }^{1}$ und Institut für Medizinische Statistik und Dokumentation (Direktor: Prof. Dr. H. Fassl) der \\ Medizinischen Universität zu Lübeck
}

\begin{abstract}
Die Einflüsse der sich ändernden Bevölkerungsstruktur auf die Entwicklung der stationären Fallzahlen und die Summe der Pflegetage in den Teilgebieten der Inneren Medizin wurden anhand der Fallstruktur des Zentrums Innere Medizin der Medizinischen Universität zu Lübeck und der Prognosen zur Bevölkerungsentwicklung des Statistischen Bundesamtes abgeschätzt. Bei gleichbleibenden Randbedingungen ist bis zum Jahre 2005 mit einem jährlichen Anstieg der internistischen Fallzahlen um $0,9 \% \mathrm{zu}$ rechnen. Die Zunahme wird für Erkrankungen der Gefäße, des Herzens, des Atmungssystems und bösartige Neubildungen überdurchschnittlich hoch, für Erkrankungen der Niere und des Verdauungssystems dagegen unterdurchschnittlich ausfallen. Vergleichende Berechnungen auf der Basis der Bundesstatistik der Krankenhausfälle der Ortskrankenkassen bestätigen im wesentlichen diese Ergebnisse. Ergänzend zeigen sie für die Summe aller nicht-pädiatrischen Fachgebiete eine nur geringe Zunahme der Fallzahlen um insgesamt $3 \%$ bis zum Jahre 2005 .

\section{Effects of demographic changes on in-patient numbers and days of hospital stay in the field of internal medicine}

Using case categories at the Centre for Internal Medicine of the Medical University at Lübeck and prognostications of future population trends by the German Federal Statistical Office, likely future numbers of in-patients and days of hospital stay were calculated. Assuming other conditions remaining constant, an annual rise of $0.9 \%$ of medical cases is to be expected until the year 2005. An increase above this average is expected for cases of cardiovascular and respiratory disease and of malignant neoplasms, while it will be below average for renal or gastrointestinal diseases. These data are similar to comparable projections made, on the basis of Federal statistics, of hospital cases registered with local health insurance schemes. In addition they indicate an increase of $3 \%$ for all non-paediatric admissions up to 2005 .
\end{abstract}

In den nächsten Jahrzehnten sind erhebliche Änderungen der Bevölkerungsstruktur in der Bundesrepublik zu erwarten. Es ist damit zu rechnen, daß die Anzahl junger Menschen abnehmen (Geburtenrückgang), die Zahl älterer Bürger dagegen erheblich zunehmen wird, bedingt zum Teil durch verlängerte Lebenserwartung, Älterwerden der »geburtenstarken Jahrgänge« und Ausgleich der durch die historische Entwicklung zwischen 1914 und 1950 besonders im männlichen Bereich verursachten Verluste $(16,17,19)$.

Das Jahresgutachten 1987 des Sachverständigenrates für die Konzertierte Aktion im Gesundheitswesen (19) enthält eine Abschätzung der Auswir-

Dtsch. med. Wschr. 114 (1989), 368-377

(c) Georg Thieme Verlag Stuttgart - New York kungen dieser demographischen Veränderungen auf die Fallzahlentwicklung aller Krankenhausfälle insgesamt ohne Aufgliederung nach Fachgruppen. Da die Altersstruktur der Patienten je nach Fachgebiet in unterschiedlicher Weise von der Bevölkerungsstruktur abweicht, sind jedoch differente Entwicklungen mit entsprechenden Folgen für die Struktur der medizinischen Versorgungseinrichtungen zu erwarten. Die Altersstrukturveränderungen beeinflussen daneben auch epidemiologische Parameter wie Geschlechtsverhältnis und Inzidenz von Erkrankungen (24). Sie beeinträchtigen damit die Vergleichbarkeit aller in klinischen

\footnotetext{
${ }^{\prime}$ Das Zentrum für Innere Medizin umfaßt die Klinik für Innere Medizin (Direktor: Prof. Dr. P. C. Scriba), Klinik für Angiologie und Geriatrie (Direktor: Prof. Dr. R. Schütz), Klinik für Kardiologie (Direktor: Prof. Dr. K. W. Diederich) und Klinik für Psychosomatik und Psychotherapie (Direktor: Prof. Dr. H. Feiereis).
} 
Untersuchungen erhobenen epidemiologischen Daten, welche die Alters- und Geschlechtsstruktur unberücksichtigt lassen.

Im folgenden soll der Einfluß der demographischen Veränderungen auf die Entwicklung der stationären Behandlungsfälle und der Pflegetage vor allem im Bereich der internistischen Teilgebiete untersucht und die Beeinflussung gebräuchlicher epidemiologischer Parameter verdeutlicht werden. Die Untersuchung wurde in zwei parallelen Ansätzen auf der Basis der Diagnosestatistik des Zentrums Innere Medizin (ZIM) der Medizinischen Universität zu Lübeck (MUL) einerseits und der Krankheitsartenstatistik der Allgemeinen Ortskrankenkassen (AOK) andererseits durchgeführt. Zur Abschätzung der Bevölkerungsentwicklung wurden Modellrechnungen des Statistischen Bundesamtes herangezogen.

\section{Methoden}

Daten der MUL. Seit dem 1.1.86 werden die Entlassungsdiagnosen aller Patienten des Zentrums Innere Medizin (ZIM) nach dem »Lübecker Diagnoseschlüssel« (ICD-L) erfaßt. Über diesen weitgehend zur Internationalen Klassifikation der Krankheiten, 9. Revision (ICD-9), kompatiblen Diagnoseschlüssel und das Vorgehen bei der Datenerfassung wurde an anderer Stelle ausführlich berichtet $(13,14)$. Die Kodierung von einer Hauptdiagnose und bis zu sechs Nebendiagnosen erfolgt durch den behandelnden Arzt bei Abfassung des Arztbriefes. Die Daten werden im Institut für Medizinische Statistik und Dokumentation weiterverarbeitet und standen dort für eine Auswertung mit Hilfe des Statistikpakets SPSS ${ }^{\circledR}$ zur Verfügung (auf einem Rechner DEC $V_{A X}{ }^{\boxplus 1 / 750)}$. Weitere Datenanalysen erfolgten auf PersonalComputern mit eigenen Programmen.

Diese Studie basiert auf den 1986 aufgenommenen stationären Behandlungsfällen des ZIM. Die Fälle der internistischen Intensiv- und Aufnahmestation wurden nur berücksichtigt, wenn von dort eine Weiterverlegung auf eine andere Station des ZIM erfolgte. Die Behandlung auf Intensiv- und Normalstation zählt dabei als ein Behandlungsfall. Von den insgesamt 6404 so definierten Fällen dieses Jahrganges waren 5775 Fälle $(90,2 \%$, entsprechend mindestens 4412 Patienten) im Rahmen der Diagnosestatistik erfaßt. Von ihnen entfielen wiederum 3484 Behandlungsfälle auf Lübecker Patienten. Die mittlere Verweildauer betrug für diese Gruppe 13,7 Tage; diese Zahl liegt wegen der andersartigen Anrechnung der Intensivpflegezeiten etwas höher als die von der Klinikverwaltung berechnete mittlere Verweildauer.

\section{Für die Gruppen}

a) alle Behandlungsfälle mit Wohnsitz der Patienten in Lübeck,

b) Untergruppen von a), die anhand der Zuordnung der Fälle zu einem internistischen Teilgebiet aufgrund der Kodierung der Hauptdiagnose gebildet wurden (Zuordnung gemäß Tabelle 1),

wurde die Anzahl der Behandlungsfälle jeweils für Männer und Frauen in den Altersgruppen 0 bis unter 5 Jahre, 5 bis unter $10, \ldots .85$ bis unter 90.90 und darüber errechnet. Wegen der Vergleichbarkeit mit den offiziellen Bevölkerungsstatistiken wurde der 31.12.85 als Stichtag für die Altersbestimmung gewählt. Durch die Beschränkung auf Patienten mit Wohnsitz in Lübeck besteht ein eindeutiger Bezug zu einer nach Alter und Geschlecht definierten Grundgesamtheit. Die Einteilung der Teilgebiete (Tabelle 1) weicht teilweise von der Unterteilung der ICD-9
Tab. 1 Zuordnung der Diagnosegruppen des Lübecker Schlüssels (ICD-L) und der vergleichbaren Gruppen der ICD-9 zu den im Text erwähnten Teilgebieten

\begin{tabular}{l|lc}
\hline \multirow{2}{*}{ Kurzname } & \multicolumn{2}{|c}{ Bezeichnung des Teilgebietes } \\
& $\begin{array}{l}\text { ICD-L Bereich } \\
\text { (MUL) }\end{array}$ & $\begin{array}{l}\text { vergleichbarer ICD-9 Bereich } \\
\text { (AOK) }\end{array}$ \\
\hline Malignome & bösartige Neubildungen \\
& $140-206$ & $140-208$ \\
Herz & Krankheiten des Herzens \\
& $390-397$ & $390-398$ \\
Gefäße & $+410-429$ & $+410-429$ \\
& Krankheiten der Arterien, Venen und Lymphgefäße \\
Atmung & $430-457$ & $430-459$ \\
& Krankheiten der Atmungsorgane \\
Verdauung & $500-508$ & $460-519$ \\
& Krankheiten der Verdauungsorgane \\
Niere & $520-577$ & $520-579$ \\
& Krankheiten der Nieren und Harnorgane \\
& $581-599$ & $580-599$ \\
\hline
\end{tabular}

ab, um den praktischen Erfordernissen der klinischen Medizin besser gerecht zu werden. Insbesondere wurde die Gruppe der "Krankheiten des Kreislaufsystems« in Anlehnung an die Teilgebiete Kardiologie und Angiologie in »Krankheiten des Herzens« und »des Gefäßsystems« unterteilt.

Die alters- und geschlechtsspezifische Wahrscheinlichkeit $\mathrm{P}_{i j}$ des Auftretens eines Behandlungsfalles im ZIM bei gegebener Struktur der Wohnbevölkerung wurde geschätzt, indem die Anzahl der Behandlungsfälle jeder Gruppe jeweils durch die entsprechende Zahl der Einwohner Lübecks gleichen Alters (i) und Geschlechts (j) dividiert wurde. Die weiteren Berechnungen stützen sich auf Modellrechnungen des Statistischen Bundesamtes zur Bevölkerungsentwicklung in der Bundesrepublik Deutschland (Entwicklung der Gesamtbevölkerung von 1985 bis 2030, Basis: 31.12 .84 . Deutsche nach Modell I, Ausländer nach Modell C) (21). Das »Modell C« des Jahresgutachtens des Sachverständigenrates (19) entspricht diesem weitgehend. Die Grundannahmen des Modells werden dort ausführlich diskutiert.

Durch Multiplikation der Einwohnerzahlen der Bundesrepublik $\left(\mathrm{N}_{\mathrm{ij}}\right)$ am 31.12.85 nach Altersgruppen und Geschlecht mit den obengenannten Zahlen und Aufsummierung wurde die fiktive Fallzahl $\Sigma N_{i j} P_{i j}$ bestimmt, die sich unter Berücksichtigung der obengenannten Wahrscheinlichkeiten für die Bundesrepublik ergeben würde. Die umgerechnete »rohe«, das heißt auf die Gesamtbevölkerung bezogene Inzidenz $\Sigma N_{i j} P_{i j} / \Sigma N_{i j}$ der Behandlungsfälle, die der aus der Bettenbedarfsrechnung bekannten "Krankenhaushäufigkeit« (6) entspricht, und das »rohe« Geschlechtsverhältnis der Behandlungsfälle wurden ebenfalls berechnet (»direkte Methode« zur Berechnung standardisierter Morbiditätsraten [2]). Die hochgerechnete Fallzahl bzw. Inzidenz für 1985 dient als Ausgangswert $(=100 \%$ ), auf den die mit gleicher Methode berechneten Zahlen für die folgenden Jahrgänge bis zum Jahre 2030 bezogen werden. Die zu erwartende Entwicklung der Summe der Pflegetage wurde in analoger Weise, nach der obengenannten Methode, aus den alters- und geschlechtsspezifischen Werten für die Anzahl der Pflegetage (die entsprechend anstelle der alters- und geschlechtsspezifischen Fallzahlen für $\mathrm{P}_{\mathrm{i1}}$ einzusetzen sind) berechnet. Es ist zu betonen, daß es sich bei der Prognose der Pflegetagssummen um eine eigenständige Berechnung handelt. Da die Verweildauer ebenso wie die Fallzahl altersabhängig ist, ist es nicht möglich, die Summe der Pflegetage über einen für alle Altersgruppen konstanten, von der Entwicklung der 
Altersstruktur unabhängigen Faktor aus der Fallzahl zu berechnen.

Das von uns angewandte Rechenverfahren, das in der epidemiologischen Standardisierungsrechnung üblich ist. läßt sich anschaulich wie folgt verstehen: Aus den bekannten Fallzahlen der MUL und den Einwohnerzahlen Lübecks wird zunächst für jede Altersgruppe und jedes Geschlecht (zum Beispiel 60- bis 64jährige Männer) die Anzahl der Behandlungsfälle pro Einwohner bestimmt. Anschließend kann diese »Morbiditätsziffer « mit der entsprechenden Einwohnerzahl einer anderen Bevölkerung (zum Beispiel Anzahl der 60- bis 64jährigen Männer im Jahr 2000 aus der Prognose des Statistischen Bundesamtes) multipliziert werden, um die Fallzahl zu erhalten, die für diese Gruppe bei Zugrundelegung der gleichen alters- und geschlechtsspezifischen Morbidität zu erwarten wäre. Führt man die Rechnung für alle Alters- und Geschlechtsgruppen durch, so erhält man in der Summe die unter den entsprechenden Annahmen zu erwartende Fallzahl der Gesamtbevölkerung.

Zur Abschätzung relativer Veränderungen reicht die Kenntnis der alters- und geschlechtsspezifischen Behandlungswahrscheinlichkeiten einer repräsentativen Stichprobe aus. Wird, wie in unserer Modellvorstellung, für alle Altersgruppen die tatsächliche Inzidenz mit dem gleichen Faktor a gewichtet, so entfällt der Faktor a durch Kürzen. Gleiches gilt auch für die relative Veränderung der »rohen « Inzidenz der Behandlungsfälle. Eine Totalerhebung für eine definierte Bevölkerungsgruppe, also etwa für alle Lübecker, ist somit für die Berechnung relativer Veränderungen nicht erforderlich, da nicht die absolute Inzidenz der stationären Behandlungsfälle bekannt sein muß, sondern lediglich der relative Anteil der Altersgruppen und Geschlechter an einer repräsentativen Stichprobe.

Daten der $A O K$. Aus den in der Diskussion näher erläuterten Gründen wurde die gesamte Berechnung auch mit den Daten zur stationären Morbidität aus der Krankheitsartenstatistik 1984 des AOK-Bundesverbandes (1) durchgeführt. Hierbei handelt es sich um eine zweite, parallele, zu Vergleichszwecken dienende Prognose auf der Basis anderer, von der Diagnosestatistik der MUL unabhängiger Daten. Die Statistik der AOK enthält Angaben über die »Krankenhausfälle und -tage nach Krankheits- und Altersgruppen « sowohl für die »Mitglieder (ohne Rentner) ohne Familienangehörige« als auch für »Rentner ohne Familienangehörige«. Aus beiden Tabellen wurde zunächst wiederum die altersund geschlechtsspezifische Inzidenz von Krankenhausfällen $\left(\mathrm{P}_{\mathrm{ij}}\right)$ der Mitglieder und Rentner zusammen (ohne Familienangehörige) für die verschiedenen Teilgebiete errechnet. Die Altersgruppen wurden entsprechend der AOK-Statistik nach folgendem Schema unterteilt: 15 bis unter 20,20 bis unter $25, \ldots, 70$ bis unter 75,75 und mehr. Die unter 15jährigen wurden aus den in der Diskussion genannten Gründen nicht berücksichtigt. Anschließend wurden die zu erwartenden Fallzahlen nach dem oben skizzierten Schema berechnet. Die AOK-Statistik beruht auf insgesamt 2,58 Millionen Krankenhausfällen bei 13,35 Millionen Mitgliedern (7,37 Millionen Männer, 5,98 Millionen Frauen).

Die Einteilung der Teilgebiete erfolgte wiederum nach den Angaben in Tabelle 1. Wegen der hohen Kompatibilität zwischen dem Lübecker Schlüssel und der ICD-9 sind die in der Tabelle angeführten Teilgebietseinteilungen nach beiden Systemen weitgehend vergleichbar. Auf (geringe) Unterschiede wird in der Diskussion der Ergebnisse eingegangen.

\section{Ergebnisse}

Abbildung 1 zeigt die Altersstruktur aller Behandlungsfälle $(\mathrm{n}=3484)$ des ZIM für die Gruppe der Lübecker Patienten. In Abbildung 2 ist oben der Anteil aller Fälle an der jeweiligen Gruppe der Wohnbevölkerung Lübecks dargestellt. Der Anteil der Behandlungs-

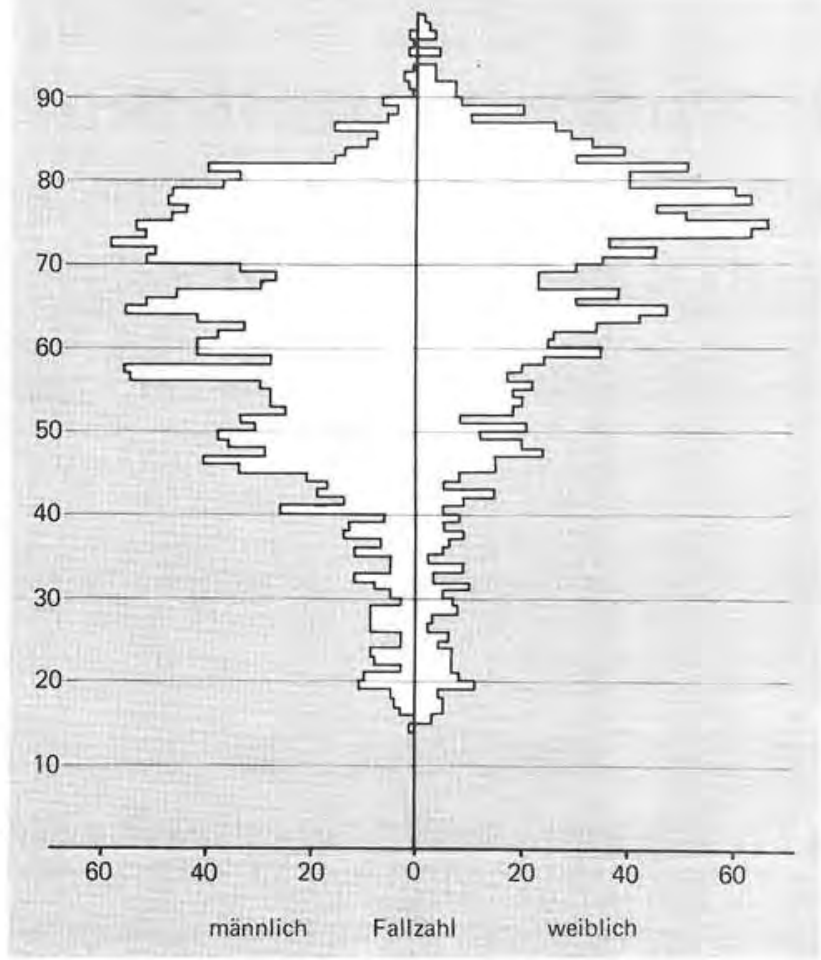

Abb. 1 Altersstruktur aller Behandlungsfälle der 1986 aufgenommenen Lübecker Patienten des Zentrums Innere Medizin. Stichtag für die Berechnung des Lebensalters ist der 31.12.85.
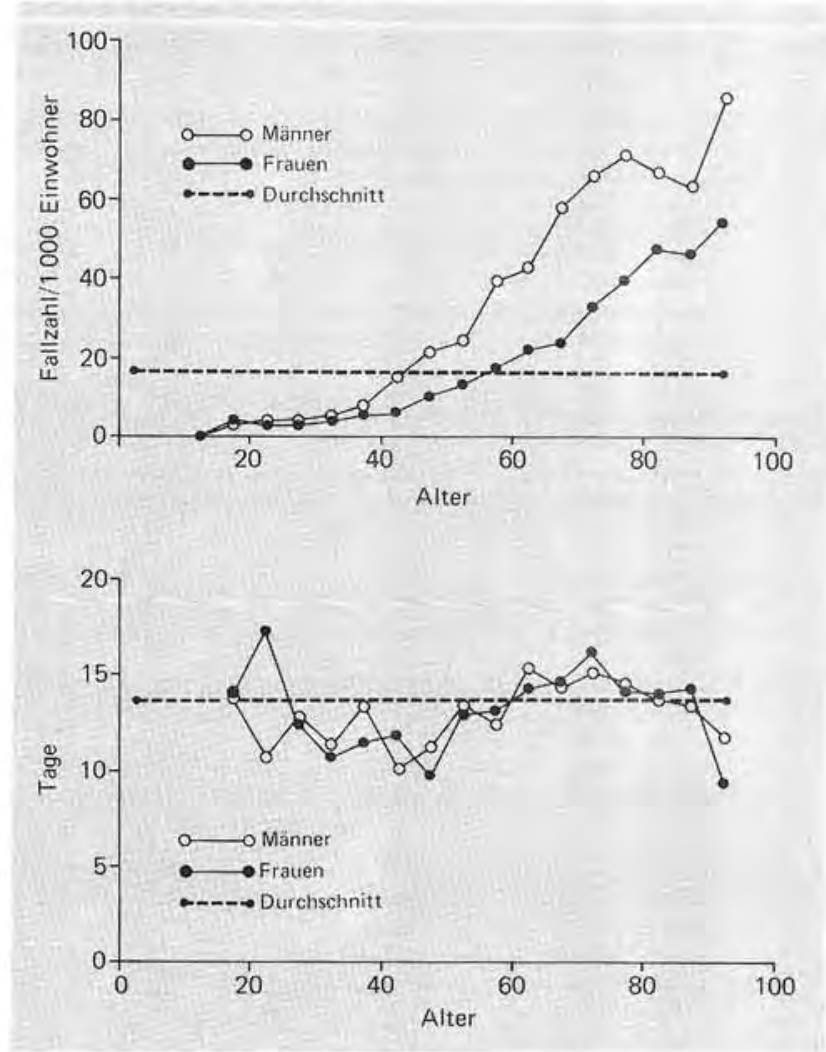

Abb. 2 Oben: alters- und geschlechtsspezifische Inzidenz aller Behandlungsfälle der 1986 aufgenommenen Patienten des Zentrums Innere Medizin der MUL, bezogen auf die Lübecker Wohnbevölkerung. Die Durchschnittslinie markiert den bei Gleichverteilung zu erwartenden Wert. Unten: Verteilung der mittleren Verweildauer in Abhängigkeit vom Lebensalter. 
Tab. 2 Aufgrund der demographischen Veränderungen zu erwartende Entwicklung der Fallzahlen auf der Basis der Fallstruktur des Zentrums Innere Medizin der MUL. Für die Prognose wurde jeweils die spezifische Alters- und Geschlechtsstruktur aller internistischen Fälle bzw. der Fälle jedes einzelnen Teilgebietes (definiert aufgrund der Hauptdiagnose nach Tabelle 1) berücksichtigt. Alle Angaben beziehen sich auf den jeweiligen Ausgangswert von 1985 (= $100 \%$ ). In der Tabelle ist zusätzlich die Veränderung des Geschlechtsverhältnisses (fallbezogen) und die relative Anderung der »rohen«, das heißt auf die jeweilige Gesamtbevölkerung bezogenen Inzidenz im Vergleich zum jeweiligen Ausgangswert von 1985 dargestellt

\begin{tabular}{l|l|l|l|l|l|l|l|l|l}
\hline Jahr & insgesamt & Herz & GefäBe & Malignome & Atmung & Verdauung & Niere & Sonstige \\
\hline $\begin{array}{l}\text { Männer } \\
\text { Fallzahl }\end{array}$ & 1866 & 624 & 331 & 187 & 178 & 139 & 88 & 319 \\
\hline 1985 & 100,0 & 100 & 100 & 100 & 100 & 100 & 100 & 100 \\
1990 & 104,9 & 105 & 105 & 108 & 106 & 101 & 105 & 104 \\
1995 & 112,6 & 115 & 114 & 114 & 115 & 106 & 108 & 109 \\
2000 & 119,9 & 121 & 122 & 125 & 122 & 113 & 117 & 115 \\
2005 & 126,8 & 128 & 133 & 133 & 131 & 116 & 118 & 119 \\
2010 & 131,2 & 134 & 139 & 136 & 134 & 118 & 126 & 122 \\
2015 & 133,6 & 138 & 143 & 138 & 135 & 113 & 133 & 122 \\
2020 & 134,1 & 138 & 146 & 138 & 137 & 111 & 133 \\
2025 & 133,4 & 137 & 145 & 139 & 139 & 108 & 130 \\
2030 & 130,7 & 132 & 145 & 139 & 139 & 104 & 128 \\
\hline
\end{tabular}

\begin{tabular}{|c|c|c|c|c|c|c|c|c|}
\hline $\begin{array}{l}\text { Frauen } \\
\text { Fallzahl }\end{array}$ & 1618 & 391 & 223 & 195 & 135 & 105 & 86 & 483 \\
\hline 1985 & 100,0 & 100 & 100 & 100 & 100 & 100 & 100 & 100 \\
\hline 1990 & 102,1 & 102 & 102 & 103 & 101 & 102 & 104 & 102 \\
\hline 1995 & 104,1 & 106 & 103 & 105 & 103 & 103 & 103 & 103 \\
\hline 2000 & 105,6 & 108 & 107 & 106 & 99 & 107 & 102 & 105 \\
\hline 2005 & 106,7 & 109 & 108 & 109 & 101 & 107 & 104 & 105 \\
\hline 2010 & 108,1 & 112 & 110 & 111 & 104 & 107 & 107 & 105 \\
\hline 2015 & 109,2 & 114 & 113 & 112 & 106 & 110 & 107 & 104 \\
\hline 2020 & 109,3 & 115 & 113 & 112 & 108 & 108 & 103 & 104 \\
\hline 2025 & 108,1 & 116 & 113 & 108 & 103 & 106 & 100 & 104 \\
\hline 2030 & 106,6 & 115 & 114 & 106 & 99 & 104 & 98 & 102 \\
\hline
\end{tabular}

\begin{tabular}{|c|c|c|c|c|c|c|c|c|}
\hline \multicolumn{9}{|c|}{ Summe Männer und Frauen } \\
\hline Fallzahl & 3484 & 1015 & 554 & 382 & 313 & 244 & 174 & 802 \\
\hline 1985 & 100,0 & 100 & 100 & 100 & 100 & 100 & 100 & 100 \\
\hline 1990 & 103,6 & 104 & 104 & 105 & 104 & 101 & 105 & 103 \\
\hline 1995 & 108,9 & 112 & 110 & 110 & 110 & 105 & 106 & 106 \\
\hline 2000 & 113,7 & 117 & 116 & 115 & 112 & 110 & 110 & 110 \\
\hline 2005 & 117,9 & 121 & 124 & 121 & 118 & 112 & 112 & 111 \\
\hline 2010 & 121,0 & 126 & 128 & 123 & 122 & 113 & 117 & 112 \\
\hline 2015 & 122,9 & 130 & 131 & 125 & 123 & 111 & 121 & 112 \\
\hline 2020 & 123,2 & 130 & 133 & 125 & 125 & 110 & 119 & 111 \\
\hline 2025 & 122,3 & 130 & 133 & 124 & 124 & 107 & 116 & 111 \\
\hline 2030 & 120,2 & 126 & 133 & 123 & 123 & 104 & 113 & 108 \\
\hline \multicolumn{9}{|c|}{ Geschlechtsverhältnis Männer : Fraven } \\
\hline 1985 & $1,28: 1$ & $1,83: 1$ & $1,66: 1$ & $1,04: 1$ & $1.41: 1$ & $1,49: 1$ & $1,09: 1$ & $0,73: 1$ \\
\hline 2000 & $1.45: 1$ & $2.05: 1$ & $1,90: 1$ & $1,22: 1$ & $1,73: 1$ & $1,57: 1$ & $1,24: 1$ & $0,80: 1$ \\
\hline 2015 & $1,56: 1$ & $2,22: 1$ & $2,11 \div 1$ & $1,28: 1$ & $1,81: 1$ & $1,53: 1$ & $1,36: 1$ & $0,85: 1$ \\
\hline 2030 & $1,57: 1$ & $2,11: 1$ & $2,13: 1$ & $1,36: 1$ & $1,98: 1$ & $1,49: 1$ & $1,42: 1$ & $0,84: 1$ \\
\hline \multicolumn{9}{|c|}{ Veränderung der »rohen« Inzidenz der Behandlungsfälle } \\
\hline 1985 & 100 & 100 & 100 & 100 & 100 & 100 & 100 & 100 \\
\hline 2000 & 115 & 118 & 118 & 117 & 114 & 112 & 111 & 111 \\
\hline 2015 & 136 & 143 & 145 & 138 & 135 & 123 & 133 & 123 \\
\hline 2030 & 153 & 160 & 170 & 156 & 156 & 133 & 145 & 138 \\
\hline
\end{tabular}


fälle nimmt mit höherem Alter erheblich zu. Das gilt in unterschiedlicher Ausprägung auch für die Teilgebiete (nicht abgebildet). Die im unteren Teil angegebenen Werte der mittleren Verweildauer zeigen etwas höhere Werte für die Altersgruppen von 60 bis 90 Jahren. Daraus ergibt sich für die Summe der Pflegetage eine etwas stärkere Altersbetonung als für die Fallzahlen.

Tabelle 2 enthält die Ergebnisse der prognostischen Berechnungen auf der Basis der Behandlungsfälle des ZIM, Angaben über die Veränderung des fallbezogenen Geschlechtsverhältnisses für die einzelnen Teilgebiete sowie die relativen Veränderungen der »rohen«, das heißt auf die Gesamtbevölkerung bezogenen Inzidenz. Letztere steigt infolge der zunehmenden Fallzahlen bei gleichzeitig insgesamt rückläufigen Einwohnerzahlen stärker an als die Fallzahlen selbst. Ausgehend von der jeweils spezifischen Alters- und Geschlechtsstruktur aller internistischen Fälle bzw. der Fälle bestimmter Teilgebiete wurde die voraussichtliche Entwicklung im Vergleich zum jeweiligen Ausgangswert von 1985 berechnet. Abbildung 3 zeigt die Ergebnisse der entsprechenden Berechnungen für die Summe der Pflegetage auf der Basis des ZIM. In Abbildung 4 ist die Fallzahlentwicklung dargestellt, wie sie sich auf der Basis der AOK-Statistik ergibt.

Die Prognose auf der Basis der Lübecker Zahlen ergibt, daß, gleichbleibende Versorgungsstrukturen vorausgesetzt, in der stationären internistischen Versorgung bis zum Jahr 2020 mit einem Anstieg der Fallzahlen um $23 \%$ zu rechnen ist. Bei Männern ist diese Entwicklung wegen der besonderen demographischen Verhältnisse und der höheren Morbidität mit $34 \%$ ausgeprägter als bei Frauen ( $9 \%$ ). Bei den Männern findet sich der höchste relative Zuwachs bei den Gefäßerkrankungen (46\%), den Erkrankungen der Atmungsorgane (39\%), den Malignomen (39\%) und den Erkrankungen des Herzens ( $38 \%$ ), also bei den Teilgebieten, die schon jetzt den größten Teil der männlichen Behandlungsfälle umfassen (zusammen $70,7 \%$ ). Bei den Frauen ist bei einem insgesamt vergleichsweise moderaten Anstieg ein überdurchschnittlicher Zuwachs bei Erkrankungen des Herzens (16\%), Gefäßerkrankungen (14\%), Malignomen $(12 \%)$ und Erkrankungen der Verdauungsorgane $(10 \%)$ zu erwarten.

Für alle Teilgebiete gilt, daß die $\mathrm{Zu}$ wachsrate der Fallzahlen von 1990 bis 2005, also schon in naher Zukunft und damit in einem Zeitraum, für den die Prognose vergleichsweise zuverlässig ist, am höchsten sein wird (im Mittel für alle internistischen Fälle jährlich 1,3\% bei Männern, 0,3\% bei Frauen; 0,9\% insgesamt). Bei weiter unveränderten Rahmenbedingungen würde zwischen den Jahren 2010 und 2025 für fast alle Teilgebiete bei nur noch geringen Veränderungen der Fallzahlen das Maximum erreicht, dem meist ein leichter, aber bereits erkennbarer Rückgang im Jahre 2030 folgt.
Die Entwicklung der Summe der Pflegetage weicht insgesamt nur wenig von der Entwicklung der Fallzahlen ab. Bis zum Jahre 2020 errechnet sich ein nur um.1,6\% größerer Anstieg. Für einzelne Teilgebiete sind aber die Abweichungen besonders bei den männlichen Patienten erheblich. In der Summe (Männer und Frauen) fällt vor allem die um $5 \%$ größere Zunahme bei Erkrankungen der Atmungsorgane auf.

Aus den in der Diskussion erörterten Gründen wurde zu Vergleichszwecken eine Prognose auf der Basis der Krankheitsartenstatistik der Ortskrankenkassen berechnet, die allerdings nicht fachabteilungsspezifisch ist. Bezogen auf alle Diagnosen ergibt sich hier für alle (nicht-pädiatrischen) AOK-Fälle ein Anstieg der Fallzahlen bei den Männern bei gleichzeitiger Abnahme der Fallzahl der Frauen ab 1990. Bis zum Jahre 2005 ergibt sich aus diesen gegenläufigen Entwicklungen in der Summe eine Zunahme um 3,1\%. Die Zahl stimmt gut überein mit den Ergebnissen ähnlicher Berechnungen im Jahresgutachten (19), die für die Zeit von 1984 bis 2000 eine Zunahme der Krankenhausfälle um etwa 4\% ergaben. Die Zahlen sind selbstverständlich nicht übertragbar auf die ausschließlich internistischen Fälle in Tabelle 2.

Bei den Erkrankungen des Herzens der AOK-Patienten dürfte der Anteil internistischer Fälle sehr hoch sein, da entsprechende (Haupt-)Diagnosen in anderen Fachbereichen, abgesehen von der Thoraxchirurgie, nur selten auftreten. Hier zeigt sich eine sehr gute Übereinstimmung zwischen den aus der ZIM-Statistik und den aus der AOK-Statistik abgeleiteten Daten. Die Werte des maximal zu erwartenden Fallzahlanstiegs differieren insgesamt um weniger als $1 \%$.

An der Behandlung der Gefäßerkrankungen sind neben der Inneren Medizin auch die Gebiete Chirurgie, Dermatologie (zum Beispiel venöse Insuffizienz), Neurologie (zum Beispiel Apoplexie) und andere beteiligt. Zu einem nicht unerheblichen Teil (etwa 10\%) sind in der AOK-Statistik auch Erkrankungen der ICDGruppen 458 (Hypotonie) und 459 (sonstige Affektionen des Kreislaufsystems, unter anderem akute Blutung verschiedener Genese) enthalten, die im Gegensatz zu den arteriellen Verschlußkrankheiten auch jüngere Patienten in erheblichem Maße betreffen. Diese Symptome, die einer Vielzahl von Krankheitsbildern ohne Bezug zu Gefäßerkrankungen zugehören, rechnen wir nicht zum klinischen Teilgebiet Angiologie, so daß sie in der ZIM-Statistik nicht berücksichtigt sind. Eine Abtrennung der beiden Gruppen aus der AOK-Statistik ist wegen mangelnder Detailliertheit nicht möglich. Die aufgrund der AOK-Statistik um etwa 10\% niedrigeren (aber mit $+21 \%$ bis zum Jahre 2020 immer noch gravierenden) Zuwachsraten dürften teilweise auf diese strukturellen Unterschiede zurückzuführen sein, könnten aber auch ein Hinweis darauf sein, daß wir den Zuwachs der Fallzahlen in diesem Teilgebiet auf der Basis der Patienten des ZIM etwas überschätzen. 

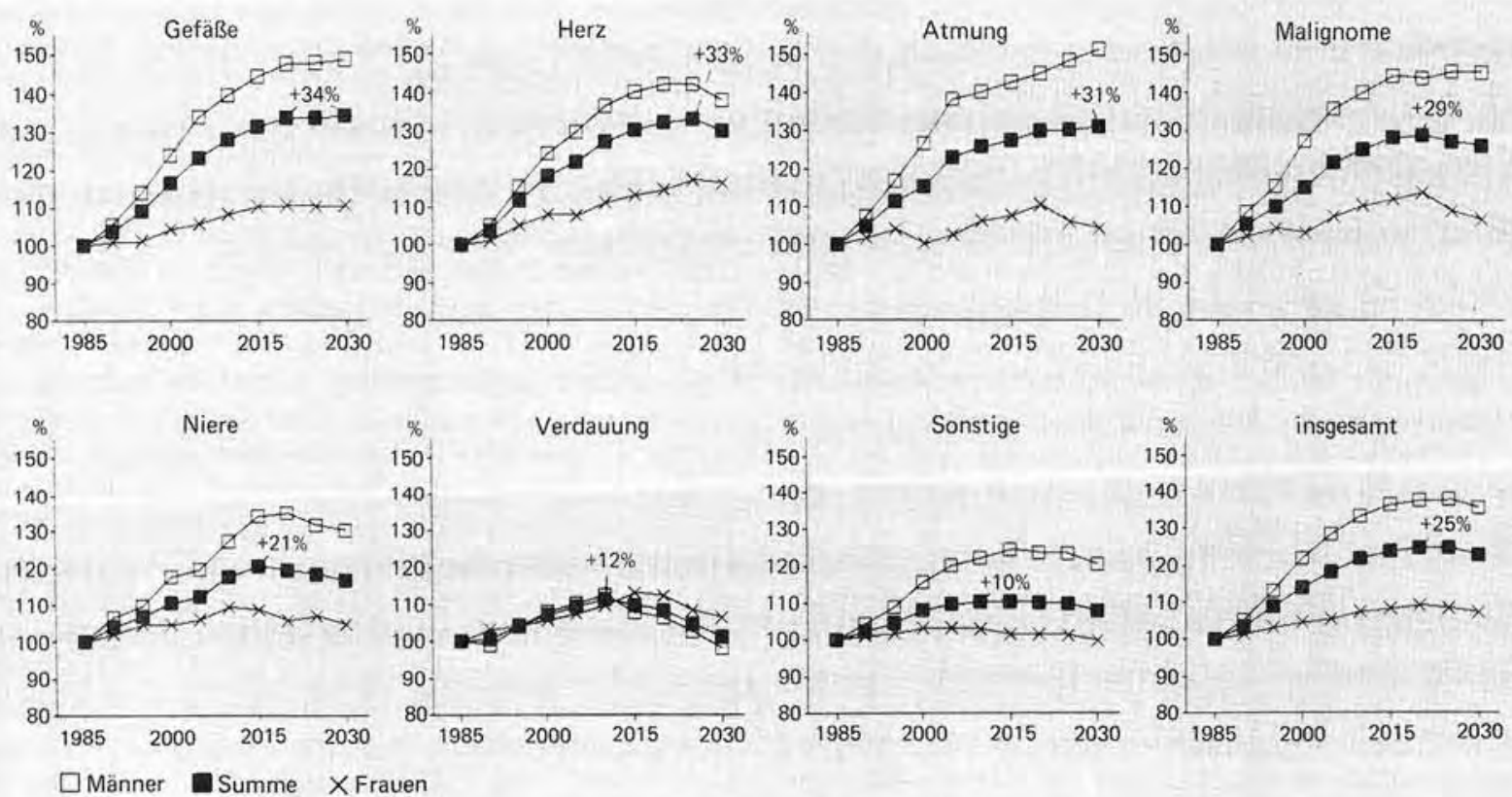

Abb. 3 Zu erwartende Entwicklung der Summe der Pflegetage unter dem Einfluß der demographischen Entwicklung im Vergleich zum jeweiligen Ausgangswert von $1985(=100 \%)$. Bei der Schätzung wurde jeweils die spezifische Alters- und Geschlechtsstruktur der Pflegetagssummen des jeweiligen Teilgebietes im Zentrum Innere Medizin der MUL berücksichtigt. Für die zu erwartenden Maxima ist jeweils die prozentuale Veränderung als Zahlenwert angegeben.
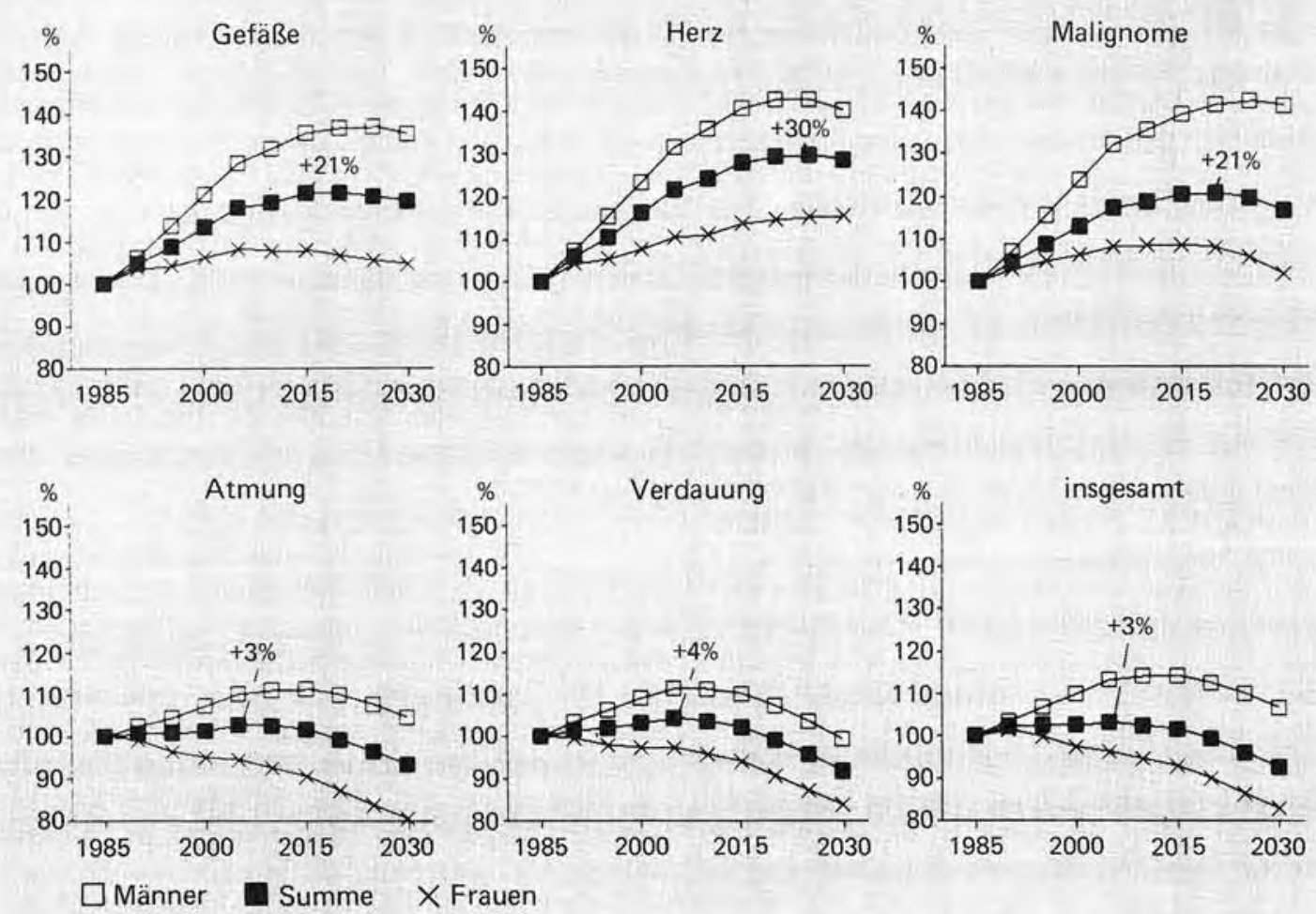

Abb. 4 Zu erwartende Entwicklung der Fallzahlen unter dem Einfluß der demographischen Entwicklung im Vergleich zum jeweiligen Ausgangswert von 1985 $(=100 \%$ ). Die Schätzung beruht auf der spezifischen Alters- und Geschlechtsstruktur der Krankenhausfälle aller AOK-Mitglieder ab dem 15. Lebensjahr in der jeweiligen Diagnosegruppe (Tabelle 1). Für die zu erwartenden Maxima ist jeweils die prozentuale Veränderung als Zahlenwert angegeben. 
In der Gruppe der Malignome umfaßt die AOK-Statistik unter anderem in erheblichem Umfang bösartige Neubildungen der Fachgebiete Gynäkologie, Urologie (beispielsweise Prostata) und anderer Gebiete. Bei den gynäkologischen Tumoren ist ein im Mittel vergleichsweise etwas niedrigeres Manifestationsalter bekannt, wodurch sich der bei weiblichen Patienten etwas geringere Anstieg der Fallzahlen auf der Basis der AOK-Statistik erklärt. Im übrigen nimmt für die Mehrzahl aller Malignome die Inzidenz in den höheren Altersgruppen deutlich zu, so daß sich trotz struktureller Unterschiede besonders für die männlichen Patienten durchaus vergleichbare Schätzwerte ergeben (Anstieg bis 2020 um 37,9vs. 41,4\% bei den Männern bzw. 24,9 vs. $20,7 \%$ insgesamt).

Die Erkrankungen der Atmungsorgane umfassen in der AOK-Statistik zu einem erheblichen Teil (35\% der Fälle) Hals-Nasen-Ohren-Erkrankungen (unter anderem Krankheiten der Nasennebenhöhlen und der Tonsillen), so daß hier wegen des überwiegend niedrigeren Manifestationsalters mit einem geringeren Anstieg zu rechnen ist. Beschränkt man allerdings die Auswertung der AOK-Fälle auf die (meist internistischen) Lungenerkrankungen (ICD 480-519, in der Abbildung nicht dargestellt), so ergibt sich auch hier eine gute Übereinstimmung mit einem Anstieg von $21,9 \%$ bis zum Jahre 2020 (gegenüber $25,0 \%$ auf der Basis ZIM). Bei den Erkrankungen der Verdauungsorgane enthält die AOK-Statistik eine Vielzahl von Krankheiten, die zu Behandlungen in der Chirurgie, in Zahnund Kieferkliniken und anderen Gebieten führen, so daß stärker abweichende Ergebnisse zu erwarten sind. Ähnliches gilt für Erkrankungen der Nieren; hier sind in der AOK-Statistik die Überschneidungen zwischen (internistischer) Nephrologie und Urologie so erheblich, daß eine Vergleichsrechnung nicht sinnvoll erschien. Für beide Gebiete dürfte die auf den Zahlen des ZIM beruhende Schätzung die Entwicklung in der Inneren Medizin besser widerspiegeln.

\section{Diskussion}

Der hohe Anteil älterer Patienten in einer internistischen Klinik ist aufgrund der unmittelbaren Erfahrung dem klinisch tätigen Arzt geläufig. In absoluten Zahlen sind am ZIM Patienten zwischen dem sechzigsten und achtzigsten Lebensjahr am stärksten vertreten. Bezieht man die Zahlen auf die entsprechenden Altersgruppen der Wohnbevölkerung, so wird erkennbar, daß der Anteil der internistischen Krankenhausfälle mit dem Lebensalter nach dem 40. Lebensjahr nahezu kontinuierlich zunimmt. Im ZIM ist der Anteil der Männer ab 65 Jahren 3,9mal, der der Frauen ab 65 Jahren 2,2mal höher, als aufgrund des Anteils der Patienten an der Bevölkerung zu erwarten wäre (korrigiertes Verhältnis Männer zu Frauen=1,8:1). Dieser Unterschied zwischen den Geschlechtern dürfte auf die höhere Morbidität der älteren Männer zurückzuführen sein, die aus epidemiologischen Studien bekannt ist (23). Sie ist im wesentlichen auf einen höheren Anteil männlicher Patienten bei gefäßbedingten Erkrankungen (Herzinfarkte, Formen der arteriellen Verschlußkrankheit) zurückzuführen.

Die genannten Ergebnisse lassen vermuten, daß eine Zunahme der älteren Bevölkerung, wie sie nach den vorliegenden Schätzungen des Statistischen Bundesamtes in den nächsten Jahren zu erwarten ist, im Bereich der Inneren Medizin eine Zunahme der Fallzahlen bewirken wird. Um eine genauere Vorstellung von der Größenordnung der allein aufgrund der demographischen Einflüsse zu erwartenden Veränderungen zu gewinnen, haben wir die relativen Veränderungen der Fallzahlen im Vergleich zu 1985 berechnet, die sich ergeben, wenn man die Krankheits- und Versorgungsstruktur des ZIM (bzw. der AOK-Versicherten) auf das Bundesgebiet überträgt und die künftige Entwicklung mit Hilfe der Bevölkerungsprognosen des Statistischen Bundesamtes hochrechnet. Die mit der Bevölkerungsvorausschätzung verbundenen Modellannahmen und prinzipiellen Probleme wurden im Jahresgutachten 1987 des Sachverständigenrates (19) und in Veröffentlichungen des Statistischen Bundesamtes (16-18) sehr ausführlich diskutiert, so daß auf Wiederholung an dieser Stelle verzichtet werden kann.

Die Aussagekraft der von uns vorgenommenen prognostischen Rechnung hängt auch davon $a b$, ob die verfügbaren Morbiditätsdaten, in diesem Fall die Alters- und Geschlechtsverteilung der Behandlungswahrscheinlichkeit, für die stationäre internistische Regelversorgung in der Bundesrepublik hinreichend repräsentativ sind. Eine allgemeine Morbiditätsstatistik, möglichst aufgeschlüsselt nach Fachgebieten, existiert nicht. Wir haben daher zwei parallele prognostische Rechnungen durchgeführt: Eine stützt sich auf ein definiertes Fallspektrum des ZIM der MUL, die andere auf die bundesweite Krankheitsartenstatistik der Ortskrankenkassen, die allerdings nicht nach Fachgebieten gegliedert ist. Dabei ermöglicht einerseits die auf die Daten des ZIM gestützte Prognose Rückschlüsse auf die Entwicklung in der Inneren Medizin, während andererseits der Vergleich mit der auf die Daten der AOK gestützten Prognose eine Beurteilung der Repräsentativität gestattet.

Die Repräsentativität des Fallspektrums der MUL, die als Universitätsklinik - je nach Spezialgebiet - einen nicht unerheblicher Teil der Patienten aus dem weiteren Umfeld zur Durchführung bestimmter, nur hier verfügbarer Leistungen aufnimmt, konnte durch Beschränkung auf Patienten mit Wohnsitz in Lübeck verbessert werden. Hier ist die Universitätsklinik zusammen mit dem Städtischen Krankenhaus Lübecks Träger der Primärversorgung. Neuzugänge werden von beiden Kliniken jeweils im täglichen Wechsel aufgenommen. Nur in Ausnahmefällen stehen spezielle Wünsche des Patienten oder des behandelnden Arztes dem entgegen. Eine besondere Auslese der Behandlungsfälle nach Alter und Geschlecht findet nicht statt. Eine Selektion hinsichtlich bestimmter Teilgebiete 
würde sich auf unsere Prognose für die jeweiligen Teilgebiete kaum auswirken, würde aber die Berechnung für die Gesamtheit der Fälle entsprechend dem größeren Gewicht des betroffenen Teilgebietes verschieben.

Obwohl infolge dieser besonderen Situation die Struktur der Behandlungsfälle der Lübecker Patienten die allgemeine Versorgungsstruktur in der Bundesrepublik recht gut widerspiegeln dürfte, wurden wegen der zu erwartenden Einwände hinsichtlich der Repräsentativität Vergleichsrechnungen anhand der Statistik der Allgemeinen Ortskrankenkassen über die Krankenhausfälle nach Alter und Geschlecht durchgeführt, die allerdings Behandlungsfälle aller Arten von Kliniken einschließt. Eine Abgrenzung der Behandlungsfälle internistischer oder anderer Kliniken ist aufgrund der Diagnose, die ja meist in mehreren Fachgebieten auftreten kann, nur sehr bedingt möglich. Die AOK-Statistik beruht ferner auf Einweisungs- und nicht auf Entlassungsdiagnosen. Auch ergeben sich trotz des Umfangs der bundesweit angelegten Statistik gewisse Einschränkungen der Repräsentativität, da aufgrund der Struktur des Krankenversicherungswesens sozial ausgewählte Fälle (unter anderem mit einem höheren Arbeiteranteil als in der Gesamtbevölkerung) und außerdem nur die Krankenhausfälle der selbst versicherten Mitglieder bzw. Rentner erfaßt werden, nicht dagegen die der Familienangehörigen. So sind nichtberufstätige Frauen unterrepräsentiert, und zuverlässige Daten für Kinder fehlen. Die Patienten unter 15 Jahren werden daher bei den Prognosen nicht berücksichtigt. Die Vergleichbarkeit mit den Daten des ZIM und der Zweck der Studie werden dadurch jedoch nicht beeinträchtigt, da diese Behandlungsfälle ohnehin den pädiatrischen Kliniken zuzurechnen sind (Anteil der Patienten unter 15 Jahren im ZIM 0,1\%).

Die mit beiden Ansätzen verbundenen methodischen Probleme sind sehr unterschiedlicher Art, so daß übereinstimmende Ergebnisse sicher nicht auf das Wirksamwerden gleicher systematischer Fehler (hinsichtlich der Gewinnung der Morbiditätsdaten) zurückzuführen sein dürften. Bei den wichtigsten der untersuchten internistischen Teilgebiete bestätigen die aus der AOK-Statistik abgeleiteten Vorhersagen der Fallzahlentwicklung weitgehend die Ergebnisse, die sich unter Zugrundelegung der Zahlen des ZIM ergeben und bestätigen damit prinzipiell die Richtigkeit der Schätzwerte. Die Abweichungen bei den Gefäßerkrankungen und besonders bei den Gruppen der Erkrankungen der Verdauungsorgane und der Nieren sind durch Unterschiede in der Diagnosestruktur bzw. das Fehlen einer Fachgebietsgliederung in der Krankheitsartenstatistik der AOK gut erklärbar.

Um den Einfluß der mit der Bevölkerungsschätzung des Statistischen Bundesamtes verbundenen Modellannahmen auf die Prognose zu veranschaulichen, wurde eine Kontrollrechnung anhand eines alternativen Modells I/D (Basis 31.12.86) des Statistischen Bundesamtes (18) durchgeführt, das etwas höhere Geburtenraten und ein anderes Wanderungsverhalten der Ausländer zugrunde legt und damit bis zum Jahr 2020 einen etwas geringeren Anteil älterer Einwohner errechnet. Die Berechnungen mit diesem Modell ergeben nur sehr geringe Unterschiede, zum Beispiel auf der Basis aller internistischen Fälle des ZIM bis zum Jahr 2020 einen Anstieg von 22,5\% gegenüber $23,2 \%$ im oben angewandten Modell; bei den Teilgebieten liegen die Abweichungen unter 1,5\%. Andere Modelle wurden vom Statistischen Bundesamt nicht veröffentlicht. Es ist aber festzustellen, daß eine Erhöhung der Geburtenrate zu einem weiteren, wegen des niedrigen Anteils junger Patienten in der Inneren Medizin aber im Prognosezeitraum nur geringfügig höheren Anstieg, eine erhöhte Sterblichkeit dagegen zu einem geringeren Anstieg der Fallzahlen führen würde.

Gegenstand dieser Studie ist die Untersuchung des Einflusses demographischer Entwicklungen auf die stationäre Morbidität. Die Untersuchung erfaßt damit zwar eine sehr wichtige Komponente der Bedarfsplanung, kann und soll aber eine vollständige Bettenbedarfsanalyse nicht ersetzen. Auf den Bedarf an Krankenhausbetten wirken sich neben der demographischen Entwicklung unter anderem auch (von der Altersstruktur unabhängige) Veränderungen der Morbidität, der Diagnose- und Behandlungsverfahren (und damit letztlich der Verweildauer) und nicht zuletzt auch gesellschaftlich bestimmte Anforderungen an die Versorgungsqualität und die Finanzierbarkeit (Kosten-Nutzen-Aspekt) sowie andere Faktoren aus, auf die hier nur kursorisch eingegangen werden kann.

Hinsichtlich der Morbiditätsveränderungen war beispielsweise für die koronare Herzkrankheit bzw. den Myokardinfarkt aufgrund von Untersuchungen der Mortalität $(4,12)$ sowie einiger autoptischer Studien (20) für verschiedene Länder (10) ein Rückgang der Morbidität vermutet worden. Eine jüngst erschienene Untersuchung über die klinische Morbidität an akutem Myokardinfarkt (8) (umfangreiche Analyse der Krankenhaus-Entlassungsdiagnosen) weist jedoch darauf hin, daß die alters- und geschlechtsspezifische Anzahl der durch Infarkte verursachten Krankenhausfälle in den USA von1974 bis 1983 bei fallender Letalität gleichblieb bzw. für einige Gruppen sogar leicht angestiegen ist. Angesichts dieser Ergebnisse ist noch unklar, ob die rückläufige Mortalität als Zeichen einer abnehmenden Morbidität durch diese wichtige Krankheitsgruppe zu verstehen ist, wobei der Rückgang der Morbidität in den stationären Behandlungsraten möglicherweise aufgrund von Artefakten (häufigere Wiederaufnahmen) nicht zum Ausdruck kommt, oder ob sich infolge erfolgreicherer Behandlungsmethoden die Überlebensraten bei gleicher Morbidität verbessert haben. Über die Folgen der AIDS-Epidemie, die sicher mit zunehmender Morbidität, besonders bei jüngeren Personen, verbunden ist, kann zur Zeit nur spekuliert werden. In den USA wird zum Beispiel für 1991 landesweit mit einem Bedarf in der Größenordnung von $2 \%$ der internistischen Betten für die Behandlung von AIDS- 
Patienten gerechnet (9). Ob eine ähnliche Entwicklung nach entsprechender Verzögerung in Deutschland zu erwarten ist, ist fraglich. Für viele andere Bereiche fehlen - insbesondere in Deutschland - zuverlässige Daten über die Morbiditätsentwicklung.

Kompliziert wird die Situation, wie auch das Beispiel der Infarktmorbidität zeigt, durch »artifizielle« Veränderungen der Fallzahlen und der Verweildauer infolge häufigerer Entlassungen und Wiederaufnahmen, zunehmender Verlegungen und anderer Faktoren, die zu einer von Morbidität und Bevölkerungsstruktur unabhängigen Komponente gegenläufiger Entwicklungen von mittlerer Verweildauer und Fallzahlen führen. Kürzere Verweildauerwerte wurden also bisher zum Teil mit Erhöhungen der Fallzahlen erkauft. Da diese Veränderungen sich nur wenig auf die Summe der Pflegetage auswirken, wurde im Jahresgutachten (19, S. 55) ein Bezug auf die Summe der Pflegetage ausdrücklich empfohlen und in unserer Studie berücksichtigt. Die Diagnosestatistik wird erst nach mehrjähriger Anwendung eine genauere Abschätzung dieser Komponente ermöglichen.

Zusammenfassend lassen die Modellrechnungen in der Bundesrepublik aufgrund der demographischen Entwicklung für alle erwachsenen Personen ( $\geqslant 15$ Jahre) und alle nicht-pädiatrischen Fachgebiete in der stationären Versorgung zunächst nur geringfügig steigende, später leicht rückläufige Fallzahlen erwarten. Im Bereich der Inneren Medizin ist jedoch schon in den neunziger Jahren wegen des Überwiegens älterer Patienten mit einem demographisch bedingten Anstieg der Fallzahlen (und des Pflegetagevolumens) um $0,9 \%$ pro Jahr zu rechnen. Bei weiter unveränderten Rahmenbedingungen dürfte die Zunahme der Fallzahlen bis etwa zum Jahre 2020 anhalten. Es ist zumindest fraglich, ob die Auswirkungen dieses Anstiegs hinsichtlich des Bettenbedarfs durch eventuelle gegenläufige Veränderungen im Sinne von abnehmender Morbidität oder Verkürzungen der Verweildauer voll kompensiert werden können. Die offensichtlich unterschiedliche Entwicklung in den verschiedenen Fachbereichen erfordert eine differenzierte Planung der Behandlungskapazitäten und läßt einen Strukturwechsel im Bereich der klinischen Versorgung unumgänglich erscheinen.

Abgesehen von der Problematik der Krankenhausplanung verdeutlichen unsere Modellrechnungen am Beispiel des einfachen Geschlechtsverhältnisses und der relativen Veränderung der »rohen«, das heißt nicht altersspezifischen Inzidenz der Behandlungsfälle auch, daß allein die zu erwartenden Änderungen der Bevölkerungsstruktur zu erheblichen Veränderungen einiger gebräuchlicher epidemiologischer Parameter führen, obwohl die alters- und geschlechtsspezifische Inzidenz, die allen Rechnungen zugrunde liegt, im Modell definitionsgemäß unverändert bleibt. Das Erkennen einer veränderten Morbidität, zum Beispiel infolge von Umwelteinflüssen oder veränderten
Lebens- und Ernährungsgewohnheiten, wird angesichts dieser Veränderungen nur möglich sein, wenn Daten über die Inzidenz einzelner Krankheitsbilder hinreichend genau nach Altersgruppen, Geschlecht (und möglichst auch weiteren Einflußfaktoren) aufgeschlüsselt vorliegen, so daß eine Umrechnung auf eine standardisierte Bevölkerungsstruktur möglich wird. In vielen Bereichen $(5,7,11)$ ist ein derartiges Vorgehen zwar durchaus üblich, bei klinischen Studien aber ist die Aufschlüsselung der Daten, oft auch wegen vergleichsweise niedriger Fallzahlen, vielfach unzureichend.

Die Diagnosestatistik nach der Bundespflegesatzverordnung, die Krankheitsartenstatistik der AOK und andere, geplante Vorhaben (3), die allerdings eher an einer ökonomischen Verwertbarkeit orientiert sind, eröffnen neue Möglichkeiten für die epidemiologische Forschung und können sich diesbezüglich ergänzen. In den USA werden detaillierte Statistiken klinischer Entlassungsdiagnosen bereits seit Jahren geführt und erfolgreich zur Bearbeitung epidemiologischer Fragestellungen eingesetzt $(8,15)$. Für derartige Vorhaben sind allerdings die in der Bundesrepublik derzeit für die Diagnosestatistik vorgeschriebene dreistellige Kodierung nur einer Diagnose und die zu grobe Altersgliederung der sogenannten L1-Statistik der Bundespflegesatzverordnung noch unzureichend. Wünschenswert wäre die Anwendung detaillierterer Verschlüsselungssysteme, wie sie beispielsweise an der Medizinischen Universität zu Lübeck und einigen anderen Kliniken bereits benutzt werden und in den USA selbstverständlich sind. Die Verwertbarkeit der bundesweiten Statistiken wird aber auch durch die nicht personen-, sondern nur fallbezogene Erfassung stark eingeschränkt, da Artefakte, zum Beispiel durch veränderte Wiederaufnahmeraten, nicht erkannt und ausgeschlossen werden können.

Wir danken dem Statistischen Bundesamt in Wiesbaden für die Überlassung der Daten zur »Entwicklung der Gesamtbevölkerung von 1985 bis 2030« sowie dem Statistischen Amt der Hansestadt Lübeck für die Überlassung der Einwohnerstatistik Lübecks. Der AOK Lübeck und dem Bundesverband der Ortskrankenkassen danken wir für die Beratung bei der Nutzung der »Krankheitsartenstatistik«.

\section{Literatur}

1 AOK Bundesverband (Hrsg.): Krankheitsartenstatistik 1984. Arbeitsunfähigkeits- und Krankenhausfälle nach Niter, Dauer, Krankheitsarten (AOK-Bundesverband: Bonn 1986).

2 Armitage, P.: Statistical Methods in Medical Research (Blackwell: Oxford 1971).

3 Brenner, G.: Brisanter Auftrag für die konzertierte Aktion. Dtsch. Ärztebl. 85 (1988), 315.

4 Cooper, R., J. Stamler, A. Dyer, D. Carside: The decline in mortality from coronary heart disease, USA, 1968-1975. J. chron. Dis. 31 (1978), 709.

5 Der Bundesminister für Jugend, Familie und Gesundheit (Hrsg.): Daten des Gesundheitswesens - Ausgabe 1985. Schriftenreihe des Bundesministers für Jugend, Familie und Gesundheit (Kohlhammer: Stuttgart 1985)

6 Femmer, H. J.: Das Krankenhaus und seine Finanzierung. Öff Gesundh.-Wes. 48 (1986), 241. 
7 Fratiglioni, L., D. Inzitari, L. Amaducci: Descriptive epidemiology of cerebrovascular disease: an update. In Ventura, A., G. Crepaldi, U. Senin (Ed.): Extracoronary Atherosclerosis, Monogr. Atheroscler. 14 (1986), 6

8 Gillum, R. F.: Acute myocardial infarction in the United States, 1970-1983. Amer. Heart J. 113 (1987), 804.

9 Gross, R.: AIDS in den Vereinigten Staaten. Aktuelle medizinische und psychosoziale Aspekte. Dtsch. Äztebl. 85 (1988), 70.

10 Kannel, W. B., T. J. Thom: Declining cardiovascular mortality. Circulation 70 (1984), 331.

11 Kaatsch, P., J. Michaelis: Modelle für Inzidenzsschätzungen bei einer Bevölkerung mit stark variierender Altersstruktur - dargestellt am Beispiel des Registers für Malignome im Kindesalter. In Überla, K., O. Rienhoff, N. Victor (Hrsg.): Medizinische Informatik und Statistik, Bd. 68. Selbmann, H.-K., K. Dietz (Hrsg.): Medizinische Informationsverarbeitung und Epidemiologie im Dienste der Gesundheit (Springer: Berlin 1988), 51.

12 Levy, R. I., Declining mortality in coronary heart disease. Arteriosclerosis 1 (1981), 312 .

13 Mansky, T., P. C. Scriba, H.-J. Friedrich, H. Fassl: Der Lübecker Diagnoseschlüssel. Klinikarzt 15 (1986), 1062

14 Mansky, T., P. C. Scriba, H. Fassl, H.-J. Friedrich: Diagnose-Verschlüsselung: wie und wozu? Dtsch. med. Wschr. 111 (1986), 1707.

15 National Center for Health Statistics: Development of the Design of the NCHS Hospital Discharge Survey. Vital and Health Statistics, Series 2, No. 39, PHS Pub. No. 1000 (Public Health Service: Washington 1970).

16 Proebsting, H.: Kein Ende in Sicht: das Geburtendefizit. Kinderarzt 15 (1984), 1571

17 Proebsting, H.: Eine neue Bevölkerungsvorausschätzung. Dtsch. Angestelltenvers. 33 (1986), 261.

18 Proebsting, H.: Trendwende in der Bevölkerungsentwicklung $1986 / 87$ ? Dtsch. Angestelltenvers. 35 (1988), 60.

19 Sachverständigenrat für die Konzertierte Aktion im Gesundheitswesen, Jahresgutachten 1987. Medizinische und ökonomische Orientierung. Vorschläge für die Konzertierte Aktion im Gesundheitswesen (Nomos: Baden-Baden 1987).

20 Solberg, L. A., J. P. Strong: Risk factors and atherosclerotic lesions. A review of autopsy studies. Arteriosclerosis 3 (1983), 187.

21 Statistisches Bundesamt, Fachserie 1, Reihe 1, Jahresband (Wiesbaden 1984).

22 Statistisches Landesamt Schleswig-Holstein: Die Bevölkerung in Schleswig-Holstein nach Alter und Geschlecht 1985. Statistische Berichte des Statistischen Landesamtes Schleswig-Holstein (Kiel 1986).

23 The Expert Panel: Report of the National Cholesterol Education Program expert panel on detection, evaluation, and treatment of high blood cholesterol in adults. Arch. intern. Med. 148 (1988), 36.

24 Weinstein, M. C., P. G. Coxson, L. W. Williams, T. M. Pass, W. B Stabson, L. Goldmann: Forecasting coronary heart disease incidence, mortality, and cost. The coronary heart disease policy model. Amer. J. publ. Hlth 77 (1987), 1417.
Dr. T. Mansky, Dipl--Psych. C. M. Erben, S. Kleinschmidt,

Prof. Dr. P. C. Scriba

Klinik für Innere Medizin

Prof. Dr. H. Fassl

Institut für Medizinische Statistik und Dokumentation

Medizinische Universität

Ratzeburger Allee 160

2400 Lübeck 